\title{
Coulisses
}

Revue de théâtre

14 | Printemps 1996

Varia

\section{Elle est là de Nathalie Sarraute}

Marie Miguet

\section{OpenEdition}

Journals

Édition électronique

URL : http://journals.openedition.org/coulisses/4834

DOI : $10.4000 /$ coulisses.4834

ISSN : 2546-9460

\section{Éditeur}

Presses universitaires de Franche-Comté

\section{Édition imprimée}

Date de publication : 1 mai 1996

Pagination : 75-76

ISSN : 1150-594X

\section{Référence électronique}

Marie Miguet, «Elle est là de Nathalie Sarraute », Coulisses [En ligne], 14 | Printemps 1996, mis en ligne le 20 mars 2019, consulté le 22 octobre 2019. URL : http://journals.openedition.org/coulisses/4834 ;

DOI : $10.4000 /$ coulisses.4834

Ce document a été généré automatiquement le 22 octobre 2019

Coulisses 


\title{
Elle est là de Nathalie Sarraute
}

\author{
Marie Miguet
}

1 «J'ai d'abord cru que c'était une histoire de 1920 et puis j'ai compris que c'était l'actualité et les camps de concentration", disait un lycéen de première ou de terminale en sortant le 2 avril d'une représentation de Elle est là donnée par le C.D.N. Personne en tout cas ne dira plus, après avoir vu cette pièce de Nathalie Sarraute, que l'auteur de Tropismes porte au théâtre des conversations d'intellectuels ou que règne sur la scène le monde des salons et des cénacles parisiens.

Dans l'œuvre dramatique, s'il n'est pas directement question de la shoah, une solution analogue est étudiée comme sont envisagées les rééducations violentes et, en cas d'échec, l'extermination de tous ceux dont la pensée n'est pas orthodoxe.

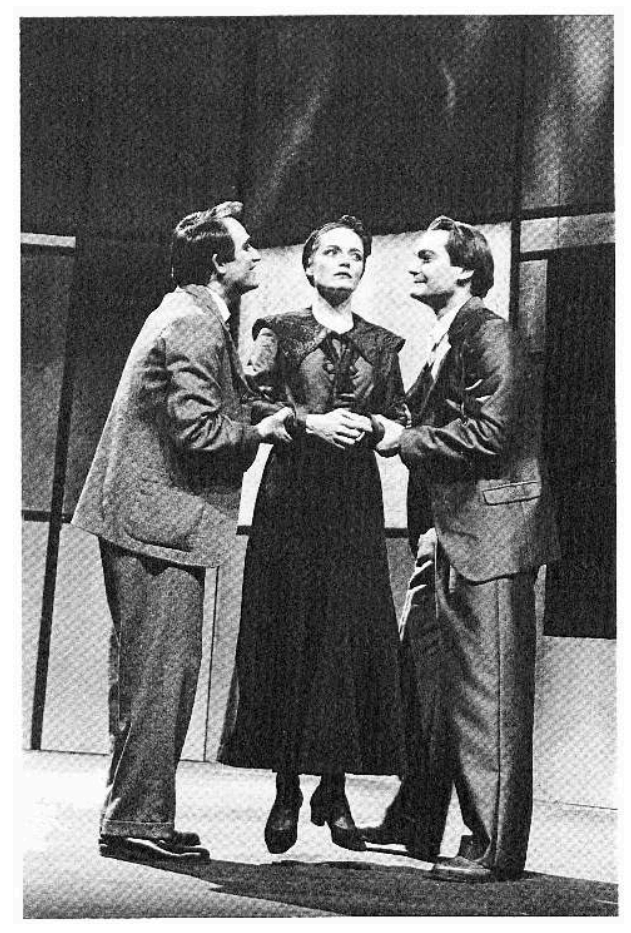
Tous les intégrismes politiques ou religieux sont visés. Un homme (H2) cherche la solution finale : comment arracher de la tête de la femme l'idée qui l'habite et qui menace la Vérité ? H2 regrette d'être seul dans la recherche de cette solution alors que dans tant de pays, c'est l'Etat qui s'en charge. A la fin de la pièce, même s'il a obtenu l'abjuration de la femme, H2 se sent vaincu : elle a eu beau rendre les armes, l'idée est là. Un jour elle sortira du lieu secret où elle est protégée.

3 Alors que dans les romans de Nathalie Sarraute, le prétexte du conflit est connu du lecteur - des fauteuils, un objet d'art - ici l'auteur pratique totalement l'abstraction. On ne sait jamais quelle est l'idée dérangeante, en quoi elle menace le système de pensée 
de H2. Au cœur de la guerre, un secret innomé. La mise en scène de Chantal Mutel est en parfait accord avec le mystère qui entoure la naissance du conflit. A sujet abstrait, décor abstrait. Un espace scénique vide; seul accessoire: un banc. Les murs sont décorés de panneaux peints qui font penser aux œuvres de Mondrian: ce sont des rectangles colorés de taille variable, avec le plus souvent des couleurs froides : bleu, vert, gris. Au début de l'action un seul panneau rouge met une couleur chaude, en accord avec le corsage rouge de la femme. Cette couleur exprime peut-être le feu, la chaleur inextinguible de la pensée qu'un Prométhée féminin a apportée aux hommes. A la fin de la pièce, un habile jeu de lumières fait disparaître le rouge, ne subsiste qu'une ambiance grise de prison et de mort. L'espace scénique devient triangulaire ; au fond, par une sorte de meurtrière filtre une lumière bleue, froide: c'est le tranchant métallique de cette Vérité dont $\mathrm{H} 2$ est désormais le seul défenseur.

A gauche du décor est ménagée une ouverture étroite, sorte de lucarne sans vitre où apparaît parfois en pleine lumière le visage de la femme ; à droite un miroir redouble les silhouettes masculines. La pièce commence par une conversation de $\mathrm{H} 2$ et $\mathrm{H} 1$ mais ce dernier manifeste peu d'intérêt pour la situation et s'éclipse vite, relayé quelque temps après par $\mathrm{H} 3$. On a une opposition entre la surface apparemment réduite réservée à l'idée dont la femme est porteuse, et les multiples reflets illusoires de la Force masculine incarnée d'abord par $\mathrm{H} 2$ et $\mathrm{H} 1$, puis par $\mathrm{H} 2$ et H3. La lumière de l'idée l'emporte; à la fin, semblant parler derrière le miroir, les partenaires masculins ont une voix qui s'estompe, qui devient mourante.

5 Un jeu de lumières et d'ombres théâtralise l'apparition sur le plateau de $\mathrm{H} 3$, surgi du fond de la salle, du milieu des spectateurs. Se détache d'abord sur la scène une ombre gigantesque : $\mathrm{H} 3$ vient apporter son secours à $\mathrm{H} 2$; cette émergence hors de l'ombre est une des trouvailles de la mise en scène; sans l'exclure, les didascalies de Nathalie Sarraute ne la préparaient pas, parlant d'un "personnage grimé en petit-bourgeois étriqué ». Si H1, qui vite se dérobe, a des vêtements qui passent inaperçus (un imperméable gris le fond dans un anonymat correct), $\mathrm{H} 2$ et $\mathrm{H} 3$ ont des tenues de ville un peu désuètes : gilet, cravate. Le tissu soyeux du costume de H2 lui donne cet aspect 1920 qui a pu tromper un instant le lycéen. A la fin, vaincu, il se dépouille de ses accessoires les plus mondains pour paraître en bras de chemise.

L'élégance de la femme est stricte; jupe longue, moulante, cheveux serrés dans un bandeau. Pas question d'essayer d'agir sur elle par le charme ou d'éveiller en elle le jeu de la coquetterie.

7 A plusieurs reprises la mise en scène de Chantal Mutel a cherché à établir une communication entre la scène et la salle: H2 interpelle les spectateurs, les rend témoins de son bon droit. Les didascalies de Nathalie Sarraute prévoient qu'une boulette lancée en l'air porte le mot " intolérance ». Seul un bruitage traduit ce passage d'un espace à un autre: $\mathrm{H} 2$ a le papier sur lui et le sort de sa poche. Sans doute sait-il depuis longtemps que cette accusation pèse sur lui : telle est, je suppose, l'intention de cette légère infidélité à la didascalie.

8 Le jeu des acteurs rend bien l'âpreté du texte de Nathalie Sarraute. La physionomie jeune et souple et le jeu de Muriel Racine, imprégné d'une rigueur toujours maitresse d'elle-même, m'ont semblé préférables à ceux de Maria Casarès (un enregistrement d'Arte m'avait permis de voir l'interprétation de la femme qu'avait donnée la grande actrice au festival d'Avignon il y a quelques années). Trop âgée, trop « monstre sacré », 
Maria Casarès avait un jeu nerveux et agressif. Celui de Muriel Racine a simplement un charme inflexible.

9 L'agressivité du jeu des acteurs masculins se tempère parfois de mondanité, de tentatives diplomatiques. Le désespoir final de $\mathrm{H} 2$ le rend presque pathétique.

Un jeu de scène m'a paru discutable. $\mathrm{H} 2$ et $\mathrm{H} 3$ usent à un moment de violence physique envers la femme, lui impriment un mouvement de ressort. La salle rit. On vérifie la formule de Bergson : le comique naît du sentiment que du mécanique est plaqué sur du vivant. Mais est-ce bien ce qu'appelle le texte? Non qu'il n'ait ses moments comiques. Mais $\mathrm{H} 2$ et $\mathrm{H} 3$ se sentent victimes d'actes de cruauté mentale. Ne doivent-ils pas réagir aussi par des sévices mentaux même s'ils portent en eux des fantasmes de meurtre et d'extermination? Plus justifié est un autre jeu de scène : H2 et H3 prennent chacun un bras de la femme, mimant ainsi son arrestation. Par cette attitude, ils la livrent à la justice, mais ils ne sont pas eux-mêmes les bourreaux.

11 Ce n'est qu'une légère réserve devant une mise en scène qui m'a paru à la fois intelligente et pleine d'invention. Un public en majorité jeune a applaudi avec enthousiasme.

\section{ANNEXES}

Approche bibliographique

Autour de Nathalie Sarraute

Les Annales Littéraires de l'Université de Besançon viennent de publier les actes d'un colloque que le Centre culturel de Cerisy-la-Salle a consacré à Nathalie Sarraute. Ont été pris en compte aussi bien les romans que l'autobiographie, les analyses critiques et le théâtre. De cette œuvre ont été proposées plusieurs lectures représentatives des principales approches de la critique contemporaine : lectures linguistiques, psychanalytiques, mythocritiques, études de l'univers imaginaire d'un écrivain qui donne une large place au corps et à une pensée en train de chercher en tâtonnant sa formulation. Philippe Lejeune, spécialiste de l'autobiographie, a eu le privilège d'avoir accès à quelques brouillons d'Enfance et peut ainsi nous livrer quelques hypothèses sur la genèse d'une écriture. 